\title{
anatomy
}

Original Article

www.anatomy.org.tr

Received: Fanuary 25, 2018; Accepted: March 10, 2018

doi:10.2399/ana.18.003

\section{Anatomical variations of the circle of Willis: evaluation with CT angiography}

\author{
Hilal Şahin, Yeliz Pekçevik \\ Department of Radiology, University of Health Sciences Tepecik Training and Research Hospital, Izmir, Turkey
}

\begin{abstract}
Objectives: The aim of this study was to investigate anatomic variants and anomalies of the circle of Willis using computed tomography angiography (CTA).

Methods: CTA images of 770 patients obtained from Tepecik Training and Research Hospital between January 2012 to January 2017 were retrospectively reviewed to identify the anatomical vascular variations of the circle of Willis.

Results: After exclusion, 751 patients (348 females, 403 males, mean age 54.6 years, range 18-90 years) were enrolled into the study. The anatomical variations related to the posterior communicating artery (PcoA) were the most common, whereas anatomical variations related to the middle cerebral artery (MCA) were the least common variations among arteries. Hypoplasia of the A1 segment was the most common (14.6\%) variation of the anterior cerebral artery (ACA) and fenestration of this artery was the least common variation (1,06\%) observed only in A1 segment. Bilateral absence of the PcoA was seen in $27.56 \%$ of the patients. Fenestration was more commonly detected in anterior communicating artery (AcoA) (10.12\%), followed by MCA $(1.06 \%), A C A(1,06 \%)$ and PCA (0.67\%). Duplication was the least common variation which was detected in MCA, AcoA and PCOA.
\end{abstract}

Conclusion: Arterial variations of the circle of Willis are not rare and can be non-invasively evaluated using CTA.

Keywords: angiography; cerebral arteries; circle of Willis; computed tomography; variation

Anatomy 2018;12(1):20-26 @2018 Turkish Society of Anatomy and Clinical Anatomy (TSACA)

\section{Introduction}

Multidetector computed tomography angiography (CTA) has proven to be a valuable tool for initial evaluation of the cerebral circulation for acute stroke and subarachnoid hemorrhage. A comprehensive CTA facilitates better visualization of cerebral vessels due to high spatial resolution and with recent advances in computed tomography (CT) technology, it has partially replaced the digital subtraction angiography. ${ }^{[1,2]}$ It does not only show the pathologies of the cerebral vasculature, but may also display clinically relevant vascular variations.

Recognizing variations of the intracranial arteries has clinical relevance. Fenestrations and duplications may predispose patients to aneurysm development. Moreover, the occlusion of an azygos or bihemispheric anterior cerebral artery (ACA) may result in ischemia of both hemispheres. Patients with fetal origin of posterior cerebral artery (PCA) and concomitant atherosclerotic disease of the carotid artery are prone to ischemic events in the PCA territory. Although the majority of normal variations have no major clinical impact, their appreciation may aid planning of the surgical and interventional procedures and may explain uncommon and unexpected findings after treatment. ${ }^{[1,3]}$

To be able to interpret the cerebral CTA correctly, we should be familiar with normal anatomy and anatomical variations of the cerebral arteries, and their cross-sectional appearances. In this study, we aimed to systematically evaluate the anatomical variations of the circle of Willis and to determine their frequency.

\section{Materials and Methods}

CTA images of 770 patients who underwent multidedector cerebral CTA in our institution from January 2012 to January 2017 were retrospectively reviewed to identify the cerebral anatomy and to determine anatom- 
ical vascular variations. Patients were referred for cerebral CTA because of known or suspected cerebral aneurysm, intracerebral hemorrhage and suspected arteriovenous malformation. We excluded all patients with a history of surgical or interventional treatment of the circle of Willis, with a cerebral occlusive disease and with poor image quality mainly due to patient movement and technical problems. The study was approved by the Tepecik Training and Research Hospital review board.

All CT examinations were performed by 2 different scanners: a 64-slice CT scanner (Aquillon 64, Toshiba Medical Systems, Tochigi, Japan) and a 128-slice CT scanner (SOMATOM Definition Edge, Siemens Healthcare, Erlangen, Germany). For venous access, an upper extremity vein (antecubital vein of the right arm) and a 20-gauge IV cannula was used. After precontrast images a total of $80-85 \mathrm{~mL}$ of contrast media with high iodine concentration $(370-400 \mathrm{mg} / \mathrm{mL})$ was injected with a flow rate of $5 \mathrm{~mL} / \mathrm{s}$, followed by a $20 \mathrm{~mL}$ saline chaser. The scanning parameters included $120 \mathrm{kV}, 225$ $\mathrm{mA}$, section thickness of $0.5 \mathrm{~mm}$ and reconstruction interval of $0.3 \mathrm{~mm}$. The scan revolution time was $0.4 \mathrm{sec}-$ onds. Data for CTA were obtained in a caudocranial direction.

In preparation of the study readings, all multidetector CTA data were transferred from the archive to a workstation (Aquarius workstation, TeraRecon, San Mateo, California, USA), via internal network connections, providing 3D postprocessing options, multiplanar image reformatting (MPR) and maximum intensity projections (MIP). We reviewed the MIP and three-dimensional volume-rendering (3D VR) images. All examinations were evaluated by two radiologists independently with respect to the anatomy of the circle of Willis. In cases of discrepancy, the CTA images were evaluated again, to reach a consensus on all variations.

We assessed the anatomy and variations of the circle of Willis and classified our findings to describe the results of our analyses. The terms of arterial variations found in our assessments are listed and explained below. ${ }^{[1,-5]}$

- Fenestration is used for an arterial lumen that divides into distinctly separate lumens with distal convergence (they may or may not share an adventitial layer).

- Duplication is used for two distinct arteries with separate origins and no distal arterial convergence.

- Absence and hypoplasia are used for absence and small size of the vessel, respectively.

- Accessory middle cerebral artery (MCA) is used for a vessel that originates from anterior cerebral artery
(ACA), courses parallel to the main MCA and supplies the anterior-inferior of the frontal lobe.

- Trifurcation of the ACA is used for presence of three ACA A2 segments. Third artery represents persistence of the median callosal artery.

- Bihemispheric ACA is used for hypoplasia of one of the ACA A2 segment. Bilateral major arterial supply is from the other, dominant $\mathrm{A} 2$ segment.

- Azygos ACA is used for single midline ACA A2 segment that represents persistence of the median callosal artery.

- Hyperplastic anterior choroidal artery (AChA) is used for prominent AChA, larger than usual.

- Infundibulum is used for cone-shaped dilatation at the origin of the posterior communicating artery (PcoA) and AChA that are smaller than $2 \mathrm{~mm}$. Those arteries arise from the apex of a cone-shaped dilatation.

- Fetal origin of the posterior cerebral artery (PCA) is used when PcoA is prominent with ipsilateral the same size or hypoplastic PCA P1 segment (partial type fetal PCA) or absent PCA P1 segment (full type fetal PCA)

\section{Results}

From 770 patients evaluated, after exclusion of the 19 patients, finally a total of 751 consecutive patients (348 females, 403 males, mean age 54.6 years, range $18-90$ years) enrolled into the study. The frequency of the detected anatomical variations was outlined in Tables 1-4. The anatomical variations related to the PcoA were the most common and anatomical variations related to the MCA were the least common variations (Figures 1 and 2). Among ACA variations, hypoplasia of the A1 segment was the most common (14.6\%) and fenestration,

Table 1

Middle cerebral artery (MCA) anatomical variations.

\begin{tabular}{lc}
\hline Anatomical variation & $\begin{array}{c}\text { Number and incidence (\%) } \\
(\mathbf{n}=\mathbf{7 5 1})\end{array}$ \\
\hline Fenestration (M1 segment) & $8(1.06)$ \\
Right & $4(0.53)$ \\
Left & $4(0.53)$ \\
\hline Accessory MCA & $7(0.93)$ \\
Right & $2(0.27)$ \\
Left & $5(0.67)$ \\
\hline Duplication & $8(1.06)$ \\
Right & $1(0.13)$ \\
Left & $7(0.93)$ \\
\hline
\end{tabular}


Table 2

Anterior cerebral artery (ACA) anatomical variations.

\begin{tabular}{lc}
\hline Anatomical variation & $\begin{array}{c}\text { Number and incidence (\%) } \\
(\mathbf{n}=\mathbf{7 5 1 )}\end{array}$ \\
\hline Hypoplasia & $110(14.6)$ \\
Right & $47(6.26)$ \\
Left & $63(8.39)$ \\
\hline Aplasia & $19(2.53)$ \\
Right & $12(1.6)$ \\
Left & $7(0.93)$ \\
\hline Fenestration* & $8(1.06)$ \\
Right & $7(0.93)$ \\
Left & $1(0.13)$ \\
\hline Trifurcation & $34(4.53)$ \\
Azygos ACA & $11(1.46)$ \\
Bihemispheric ACA & $9(1.2)$ \\
\hline
\end{tabular}

*All fenestrations were in ACA A1 segment.

Table 3

Anterior communicating artery $(\mathrm{AcO} A)$ anatomical variations.

\begin{tabular}{lc}
\hline Anatomical variation & $\begin{array}{c}\text { Number and incidence (\%) } \\
(\mathbf{n}=\mathbf{7 5 1 )}\end{array}$ \\
\hline Fenestration-duplication* & $76(10.12)$ \\
Absence & $29(3.86)$ \\
\hline
\end{tabular}

*Only one patient identified as duplication.

Table 4

Posterior communicating artery (PCOA) and posterior cerebral artery (PCA) anatomical variations.

\begin{tabular}{lc}
\hline Anatomical variation & $\begin{array}{c}\text { Number and incidence (\%) } \\
(\mathbf{n}=751)\end{array}$ \\
\hline Absent PcoA & $355(47.3)$ \\
Right & $64(8.52)$ \\
Left & $84(11.19)$ \\
Bilateral & $207(27.56)$ \\
\hline Partial type fetal origin of the PCA* & $190(25.3)$ \\
Right & $87(11.58)$ \\
Left & $57(7.59)$ \\
Bilateral & $46(6.13)$ \\
\hline Total type fetal origin of the PCA & $6(0.79)$ \\
Right & $3(0.39)$ \\
Left & $2(0.27)$ \\
Bilateral & $1(0.13)$ \\
\hline PCA Fenestration & \\
Right & $5(0.67)$ \\
Left & $3(0.39)$ \\
\hline PCoA Duplication & $2(0.27)$ \\
Right & $1(0.13)$ \\
Left & $1(0.13)$ \\
\hline
\end{tabular}

*Partial type: prominent PcoA with ipsilateral the same size or hypoplastic P1 segment of the PCA; Total type: Prominent PcoA with ipsilateral absent P1 segment of the PCA; ${ }^{\ddagger}$ PCA fenestrations were P1 segment in all patients.

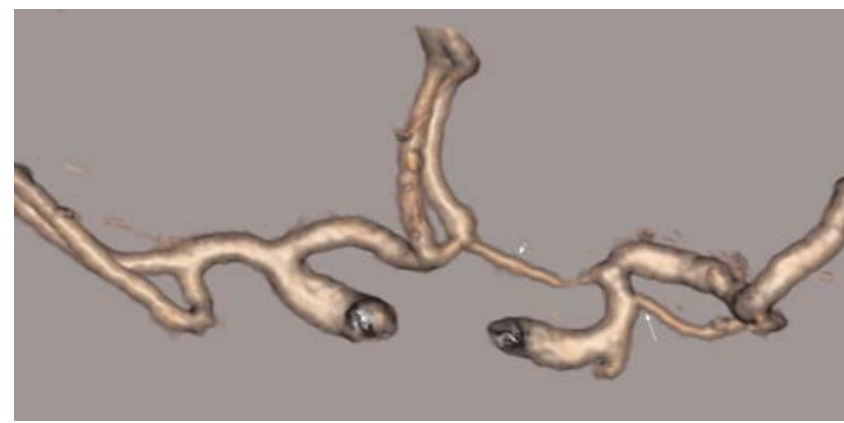

Figure 1. 3D VR image shows a duplicated MCA (long arrow). A smaller branch arises from ICA just before its terminal bifurcation and courses parallel to the main branch. Left A1 segment of the ACA is hypoplastic (short arrow). [Color figure can be viewed in the online issue, which is available at www.anatomy.org.tr]

which was observed in only A1 segment, was the least common (1.06\%) variation (Figures 3-7). Bilateral absence of the PcoA was seen in 207 of 751 patients (27.56\%) (Figure 8). Fenestration was more commonly detected in anterior communicating artery (AcoA) (10.12\%), followed by MCA (1.06\%), ACA $(1,06 \%)$ and PCA $(0.67 \%)$. Duplication was the least common variation which was detected in MCA, AcoA and PcoA.

\section{Discussion}

The reported prevalence of most of the vascular variants and anomalies is derived from cadaveric or angiographic studies. The knowledge of the segmental anatomy and the prevalence of the arterial variants is important while evaluating multidedector CTA images. We evaluated anatomical variations of the cerebral arteries in 751 patients and found that those anatomical variations are frequent and could be evaluated correctly by CTA.

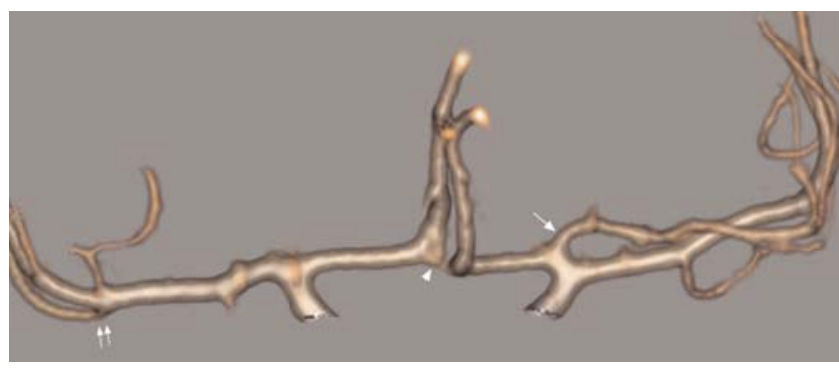

Figure 2. 3D VR image depicts an accessory MCA (arrow), a smaller branch arises from A1 segment of the ACA. There is also anterior communicating artery fenestration (arrowhead) and trifurcation of the right MCA (double arrows). [Color figure can be viewed in the online issue, which is available at www.anatomy.org.tr] 


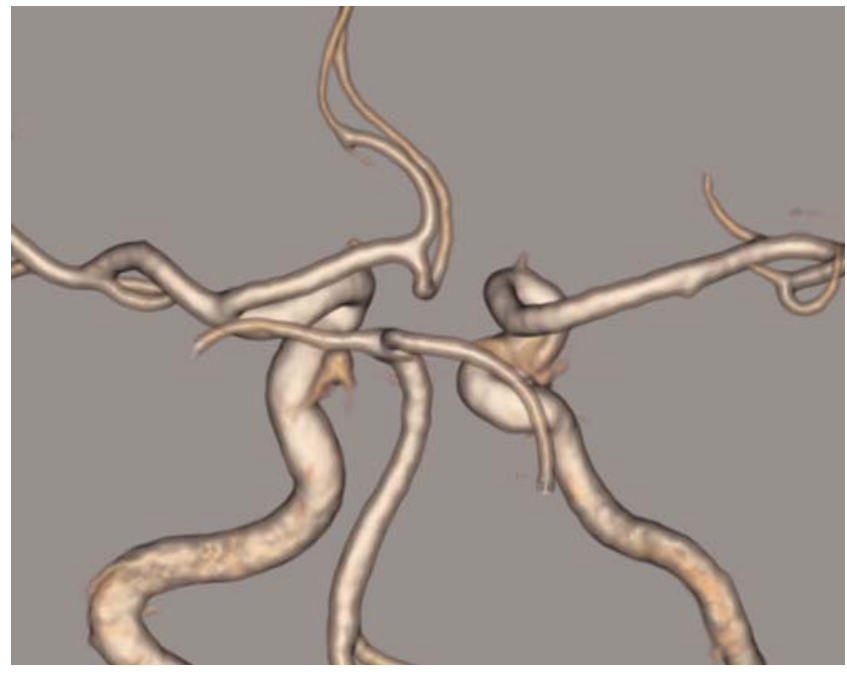

Figure 3. 3D VR image demonstrates absence of the A1 segment of the ACA. [Color figure can be viewed in the online issue, which is available at www.anatomy.org.tr]

DSA, used for visualization of the cerebral arterial system for several decades, has disadvantages in detecting cerebral artery anomalies because of the $2 \mathrm{D}$ projection of the images. Most anomalies are only visible from one specific angle, that is likely not present in the limited available projections of conventional angiography. Any desired viewing angle is possible with $3 \mathrm{D}$ imaging. Although 3D rotational angiography has higher spatial resolution than CTA, it is invasive and lack of soft tissue information. ${ }^{[6,7]}$ CTA is not affected by flow-related inhomogeneities, which is very commonly seen at MR angiography. CTA is performed in seconds, as opposed to minutes, effectively eliminating MR angiography which is limited by the patients' motion and it offers more complete intracranial coverage in less time than does MR angiography. ${ }^{[7]}$ Furthermore, CTA enables better visualization of small vessels and vascular variations. ${ }^{[8]}$

Anatomical variations of the middle cerebral artery were not found seen frequently in our study. An accessory MCA was observed in $0.93 \%$ of the patients and the frequency of accessory MCA is reported as $0.3 \%$ to $4 \%{ }^{[4]}$ Duplication of the MCA is reported as $0.2 \%$ to $2.9 \% .^{[3,4]}$ In our study it was found in $1.06 \%$ of the patients. Both variations may change arterial blood supply regions. ${ }^{[8,9]}$ These variations should be considered in evaluating ischemic lesions and clinical symptoms in stroke patients. ${ }^{[8,10]}$ The clinical significance of MCA fenestration might be due to its relationship with aneurysm formation. ${ }^{[1,12]}$ But none of our patients with MCA fenestration (8 patients, $1.06 \%)$ had an aneurysm.

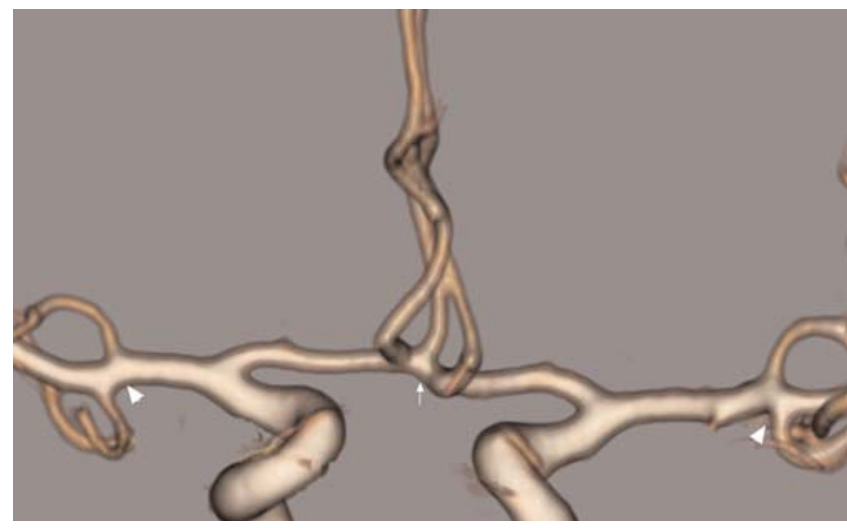

Figure 4. 3D VR image shows trifurcation of the ACA, two normal $A 2$ segments and one $A 2$ segment that arises from the anterior communicating artery (arrow). Bilateral trifurcation of MCA (arrowhead) accompanies to this anomaly. [Color figure can be viewed in the online issue, which is available at www.anatomy.org.tr]

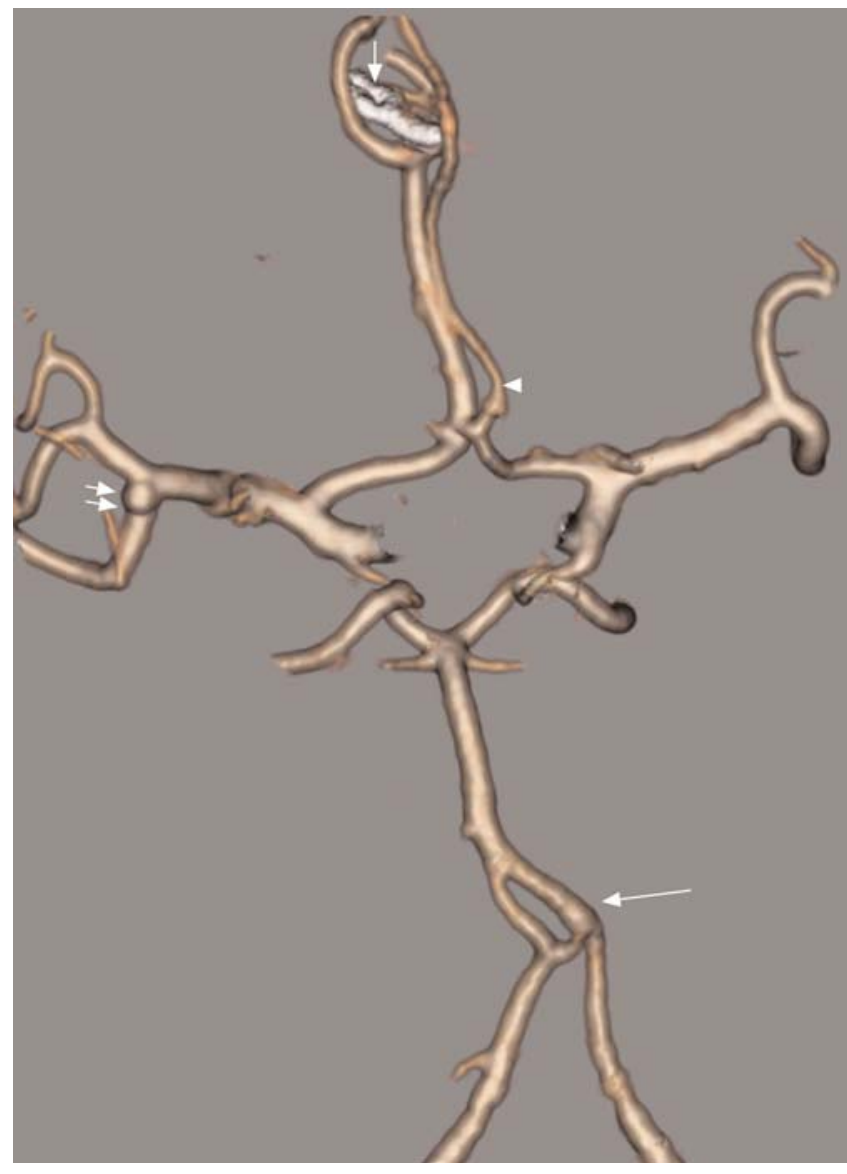

Figure 5. 3D VR image demonstrates bihemispheric ACA. Dominant A2 segment that supplies both ACA territories has operated aneurysm at the level of furcation (short arrow). The smaller caliber, non-dominant A2 segment (arrowhead) courses parallel to the dominant segment. There is small aneurysm at MCA bifurcation (double arrows). Basilar artery fenestration (long arrow). [Color figure can be viewed in the online issue, which is available at www.anatomy.org.tr] 
Anterior cerebral artery A1 segment hypoplasia and absence was found $14.6 \%$ and $2.53 \%$, respectively. The reported incidence in cadaveric studies, $10 \%$ for hypoplasia and $1-2 \%$ for absence, is similar with our findings. ${ }^{[1,3]}$ These anatomical variations cause decreased collateral supply in the event of thromboembolic disease. Two normal A2 segment with persistence of the embryonic median artery of the corpus callosum defined as ACA trifurcation. Its incidence varies from 2 to $13 \%$. $^{[3]}$ In the present study, it was found in $4.53 \%$ of the patients. Bihemispheric ACA represents one hypoplastic A2 segment with the other A2 segment provide the major blood supply to both hemispheres. The prevalence of this variation is $2-7 \%$ in cadaveric studies and we observed $1.2 \%$ of the patients in our study. ${ }^{\left[{ }^{3}\right]}$ The frequency was less than both cadaveric and angiographic studies. ${ }^{[13]}$ This anatomical variation may be related to aneurysm formation. ${ }^{[14]}$ We had only one patient with

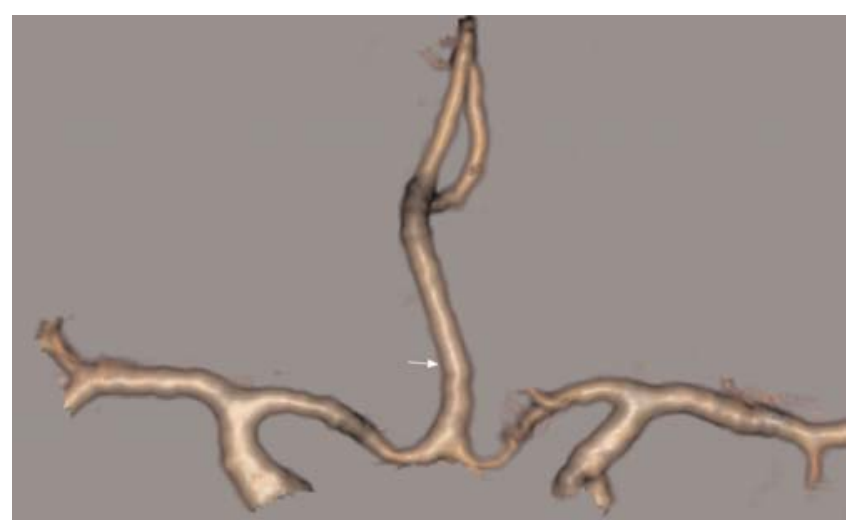

Figure 6. 3D VR image depicts azygos ACA, a single midline A2 trunk (arrow). [Color figure can be viewed in the online issue, which is available at www.anatomy.org.tr]

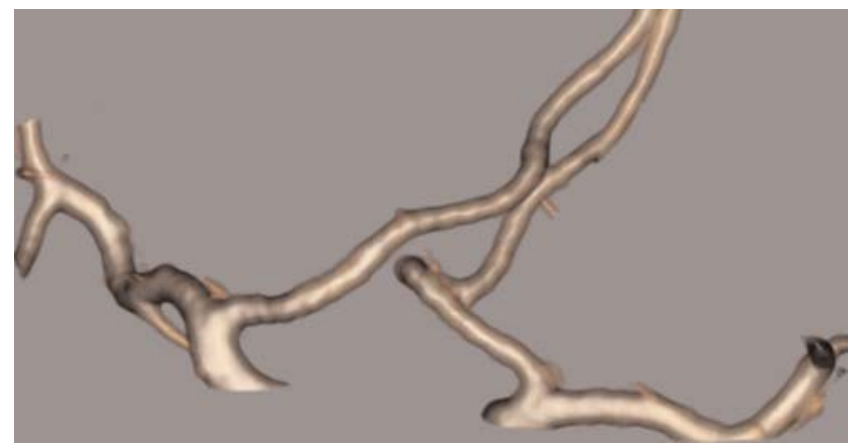

Figure 7. 3D VR image shows the absence of the anterior communicating artery. [Color figure can be viewed in the online issue, which is available at www.anatomy.org.tr] aneurysm at bifurcation of the normal A2 segment, that ruptured and was treated with surgical clips. Azygos ACA is defined as a single unpaired A2 segment that represents persistence of the median artery of the corpus callosum. The prevalence of azygos ACA is between $0.2 \%$ and $4 \%$ and it was found in $1.46 \%$ of patients in our study. Azygos ACA have been reported to be associated with many congenital anomalies like holoprosencephaly and an increased risk of aneurysm formation. ${ }^{[15]}$ Both bihemispheric and azygos ACA are clinically important anomalies and should be reported because occlusion of the ACA from whatever cause affects both hemispheres. ${ }^{[1,3]}$

Fenestration was the most frequently observed in AcoA (10.12\% of patients) in our study. The reported incidence of AcoA fenestration from anatomic, 3D angiographic and CT angiographic studies is $12-20 \%$, $5.3 \%$ and $5.32 \%$, respectively ${ }^{[16,17]}$ Anterior communicating artery is so small that recognition of its anomalies may be difficult on CTA in comparison to the cadaveric studies. On the other hand, our higher incidence when compared previous studies with angiography and CTA is probably due to the recent improvements in CT technology that allows obtaining thinner images with higher resolution. ${ }^{[6,8]}$ The clinical significance of fenestration comes from its association with the aneurysm formation. It is postulated that in addition to arterial wall weakness, the hemodynamic factors result in formation of an aneurysm at the proximal end of arterial fenestrations.

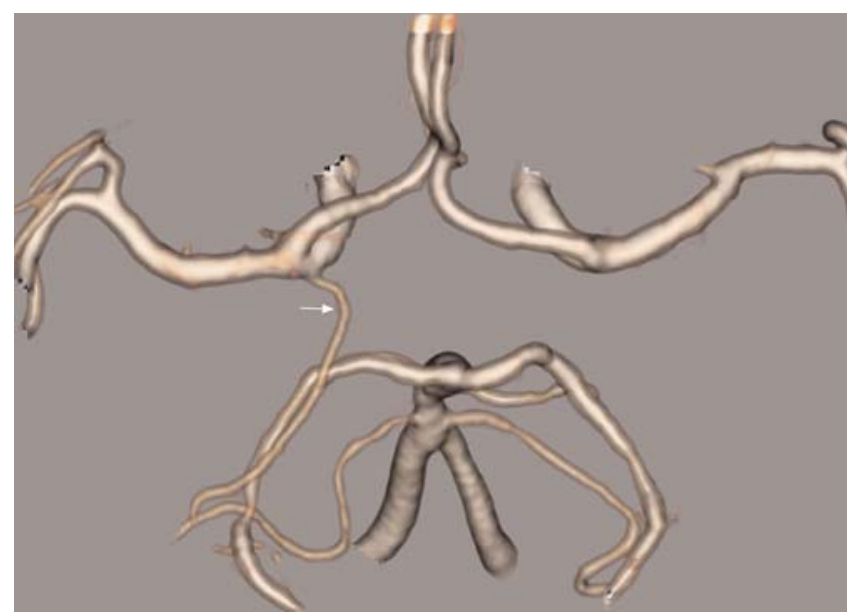

Figure 8. 3D VR image shows the absence of both posterior communicating arteries. Note that the anterior choroidal artery is prominent, so called as hyperplastic in 3D VR image (arrow). [Color figure can be viewed in the online issue, which is available at www.anatomy. org.tr] 
An absence of AcoA occurs in $5 \%$ of surgical dissections. ${ }^{[1,3]}$ In our study, the percent of absence of AcoA was $3.86 \%$, which was lower than anatomical studies in the literature.

The posterior communicating arteries are usually small and may not be depicted at CTA, but this does not necessarily mean that the arteries are absent. Hypoplasia of PCoA is seen in one fourth of MR angiograms and one third of all anatomic dissections. ${ }^{[3]}$ In our study, it was the most frequent anatomical variation and observed in $47.3 \%$ of the patients. Bilateral absence was higher than unilateral absence and unilateral absence was more common on the left side. Posterior communicating arteries are main collaterals of the circle of Willis and knowing their patency is important before any surgical and interventional procedure.

Fetal origin of the posterior cerebral artery occurs when the embryonic posterior cerebral artery fails to regress. ${ }^{[1]}$ When the prominent $\mathrm{PcoA}$ is observed with the same size or hypoplastic ipsilateral P1 segment of the PCA, it is named as partial type fetal origin of the $\mathrm{PCA}^{\left[{ }^{[18]}\right.}$ But if the ipsilateral P1 segment of the PCA is absent, it is called full or total type fetal origin of the PCA ${ }^{[1,18]}$ Prevalence of partial type from cadaveric, DSA and MR angiographic studies have wide range, 11-29\% for unilateral and $1-9 \%$ for bilateral. ${ }^{[18,19]}$ It may occur $10 \%$ on the right side and $10 \%$ on the left side and $8 \%$ bilaterally. ${ }^{[19]}$ In the present study, we observed partial type fetal origin of PCA in $25.3 \%$ of the patients, $11.58 \%$ on the right and $7.59 \%$ on the left side and $6.13 \%$ bilaterally. Total type fetal origin of the PCA is very uncommon. ${ }^{[18]}$ In our study, $0.79 \%$ of the patients had this anatomical variation. Most of them were unilateral with exception of one bilateral patient. Reporting of either type of this variant is clinically important because dominant blood supply to the occipital lobes comes from internal carotid artery and PCA territory stroke may be caused by atheromatous disease of the anterior circulation. ${ }^{[17-19]}$ Posterior cerebral artery fenestration is extremely rare ${ }^{[1,19]}$ We observed it only in five patients $(0.67 \%)$. Posterior communicating artery duplication was the least common variation in the present study with $0.13 \%$ prevalence rate which is also extremely rare.

This study also has some limitations. First of all, very small arteries may not be detected via CTA. The absence or hypoplasia of an artery was decided by the consensus of two observers in the study. Secondly, in patients with a silent occlusive arterial disease, the occluded artery could be misdiagnosed as absent. To overcome this lim- itation, contrast enhanced images were compared with the non-enhanced images.

\section{Conclusion}

According to our large study group, we believe that the arterial variations of the circle of Willis are not rare, clinically important and can be noninvasively evaluated by CTA.

\section{References}

1. Dimmick SJ, Faulder KC. Normal variants of the cerebral circulation at multidetector CT angiography. Radiographics 2009;29:102743.

2. van Rooij SB, van Rooij WJ, Sluzewski M, Sprengers ME. Fenestrations of intracranial arteries detected with 3D rotational angiography. AJNR Am J Neuroradiol 2009;30:1347-50.

3. Osborn AG. Diagnostic cerebral angiography (2nd ed). Philadelphia, PA: Lippincott Williams \& Wilkins; 1999.

4. Komiyama M, Nakajima H, Nishikawa M, Yasui T. Middle cerebral artery variations: duplicated and accessory arteries. AJNR Am J Neuroradiol 1998;19:45-9.

5. Parmar H, Sitoh YY, Hui F. Normal variants of the intracranial circulation demonstrated by MR angiography at 3T. Eur J Radiol 2005 ; $56: 220-8$.

6. Jayaraman MV, Mayo-Smith WW, Tung GA, Haas RA, Rogg JM, Mehta NR, Doberstein CE. Detection of intracranial aneurysms: multi-detector row CT angiography compared with DSA. Radiology 2004;230:510-8.

7. Pekcevik Y, Pekcevik R. Variations of the cerebellar arteries at CT angiography. Surg Radiol Anat 2014;36:455-61.

8. Nussbaum ES, Defillo A, Janjua TM, Nussbaum LA. Fenestration of the middle cerebral artery with an associated ruptured aneurysm. J Clin Neurosci 2009;16:845-7.

9. Songur A, Gonul Y, Ozen OA, Kucuker H, Uzun I, Bas O, Toktas M. Variations in the intracranial vertebrobasilar system. Surg Radiol Anat 2008;30:257-64.

10. Kiroğlu Y, Karabulut N, Oncel C, Akdogan I, Onur S. Bilateral symmetric junctional infarctions of the cerebellum: a case report. Surg Radiol Anat 2010;32:509-12.

11. de Gast AN, van Rooij WJ, Sluzewski M. Fenestrations of the anterior communicating artery: incidence on $3 \mathrm{D}$ angiography and relationship to aneurysms. AJNR Am J Neuroradiol 2008;29:296-8.

12. Reis CV, Zabramski JM, Safavi-Abbasi S, Hanel RA, Deshmukh P, Preul MC. The accessory middle cerebral artery: anatomic report. Neurosurgery 2010;66:E1217.

13. Hamidi C, Bükte Y, Hattapoğlu S, Ekici F, Tekbaş G, Önder H, Gümüs H, Bilici A. Display with 64-detector MDCT angiography of cerebral vascular variations. Surg Radiol Anat 2013;35:729-36.

14. Kashiwazaki D, Kuroda S, Horiuchi N, Takahashi A, Asano T, Ishikawa T, Iwasaki Y. Ruptured aneurysm of bihemispheric anterior cerebral artery bifurcation: case report. No Shinkei Geka 2005;33: 383-7.

15. Cinnamon J, Zito J, Chalif DJ, Gorey MT, Black KS, Scuderi DM. Aneurysm of the azygos pericallosal artery: diagnosis by MR imaging and MR angiography. AJNR Am J Neuroradiol 1992;13:280-2. 
16. Bayrak AH, Senturk S, Akay HO, Ozmen CA, Bukte Y, Nazaroglu $\mathrm{H}$. The frequency of intracranial arterial fenestrations: a study with 64-detector CT-angiography. Eur J Radiol 2011;77:392-6.

17. van der Lugt A, Buter TC, Govaere F, Siepman DA, Tanghe HL, Dippel DW. Accuracy of CT angiography in the assessment of a fetal origin of the posterior cerebral artery. Eur Radiol 2004;14: $1627-33$.

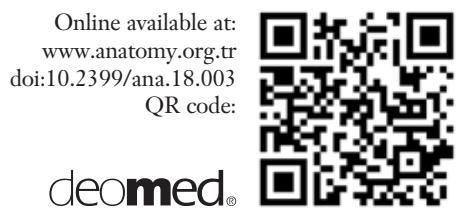

18. van Overbeeke JJ, Hillen B, Tulleken CA. A comparative study of the circle of Willis in fetal and adult life: the configuration of the posterior bifurcation of the posterior communicating artery. J Anat 1991; 176:45-54.

19. van Raamt AF, Mali WP, van Laar PJ, van der Graaf Y. The fetal variant of the circle of Willis and its influence on the cerebral collateral circulation. Cerebrovasc Dis 2006;22:217-24.

Correspondence to: Yeliz Pekçevik, MD

Department of Radiology, University of Health Sciences

Tepecik Training and Research Hospital, Izmir, Turkey

Phone: +90 2324443560 / 1660

e-mail: yelizpekcevik@yahoo.com

Conflict of interest statement: No conflicts declared.

This is an open access article distributed under the terms of the Creative Commons Attribution-NonCommercial-NoDerivs 3.0 Unported (CC BY-NCND3.0) Licence (http://creativecommons.org/licenses/by-nc-nd/3.0/) which permits unrestricted noncommercial use, distribution, and reproduction in any medium, provided the original work is properly cited. Please cite this article as: Şahin H, Pekçevik Y. Anatomical variations of the circle of Willis: evaluation with CT angiography. Anatomy 2018;12(1):20-26. 